\title{
Steroid Metabolic Process
}

National Cancer Institute

\section{Source}

National Cancer Institute. Steroid Metabolic Process. NCI Thesaurus. Code C18109.

Steroid Metabolism consists of diverse biochemical reactions that convert steroid compounds, lipids containing a hydrogenated cyclopentanoperhydrophenanthrene ring system, to metabolic derivatives for assorted uses, such as steroid hormone production and biosynthesis of cell membrane constituents. 